\title{
Treatment-Refractory Headache in the Setting of COVID-19 Pneumonia: Migraine or Meningoencephalitis? Case Report
}

\author{
Karissa N. Arca ${ }^{1}$ - Amaal J. Starling ${ }^{1}$ \\ Accepted: 17 June 2020 / Published online: 25 June 2020 \\ (C) Springer Nature Switzerland AG 2020
}

\begin{abstract}
The coronavirus responsible for the COVID-19 pandemic, SARS-2-CoV, most commonly involves the respiratory tract; however, more severe cases have been found to have multi-organ involvement, including the central nervous system. Headache has been documented as a presenting symptom of COVID-19, which may prompt neurology consultation. To date, few cases of COVID-19 meningitis have been confirmed, but it must remain in the differential diagnosis for patients with headache and fever. A 58-year-old female with multiple sclerosis on fingolimod, chronic migraine well-controlled on fremanezumab, and cerebrovascular disease presented with fevers and respiratory symptoms, as well as acute treatment-refractory headache with associated neck stiffness and transient behavioral abnormalities. Although not confirmed with cerebrospinal fluid testing, we suspect this patient had COVID-19 meningoencephalitis, highlighting the need to consider secondary headaches in patients with coronavirus infection, even in the setting of chronic migraine. We offer anecdotal treatment recommendations for acutely refractory secondary headache and guidance for the consulting neurologist during the COVID-19 pandemic.
\end{abstract}

Keywords COVID-19 $\cdot$ Coronavirus $\cdot$ Migraine $\cdot$ Headache $\cdot$ Meningitis $\cdot$ Meningoencephalitis

\section{Introduction}

In the outpatient or inpatient setting, headache may be a presenting symptom of COVID-19, with up to one third of patients experiencing neurologic symptoms at some point during the illness [1]. A recent retrospective review from Wuhan, China, found that some patients were admitted to the neurology service with chief complaints of fever and headache with delayed development of respiratory symptoms, later confirmed as COVID-19 by respiratory pathogen polymerase chain reaction (PCR) [1]. The Centers for Disease Control (CDC) have recognized that headache can be a sentinel symptom of COVID-19 [2]. A patient presenting with either new or worsening headache should prompt screening for other COVID-19 symptoms and PCR testing if the screen is positive. There are limitations to treating a headache exacerbation

This article is part of the Topical Collection on Covid-19

Karissa N. Arca

Arca.Karissa@mayo.edu

1 Mayo Clinic Arizona, 13400 E Shea Blvd, Scottsdale, AZ 85259, USA in the setting of COVID-19, some founded on evidence while others are not. Mixed reports about the use of antiinflammatory medications, particularly non-steroidal anti-inflammatories (NSAIDs) seem to have stemmed from the tweet [3] of a French neurologist cautioning against their use. Since then, the World Health Organization (WHO) initially recommended against the use of NSAIDs in COVID-19 but later retracted the recommendation [4]. The use of steroids, however, is not recommended in patients with COVID-19 by multiple health agencies including the WHO, Centers for Disease Control (CDC), and National Institutes of Health (NIH) [4-6]. Distinguishing a primary headache from a secondary headache disorder in the setting of COVID-19, as well as the treatment of each, requires the discerning knowledge and care of a neurologist.

\section{Case Presentation}

We present a case of a 58 -year-old female with multiple sclerosis on fingolimod, chronic migraine on fremanezumab, and history of cerebrovascular ischemic disease who was admitted for COVID-19 pneumonia. Neurology was consulted for worsening headache and dysphagia. A phone consultation was performed to limit exposure of the consulting service. 
The patient had been taking fremanezumab for the past 1 year with excellent efficacy. Prior preventive therapies included topiramate and onobotulinum-toxin-A. She was taking gabapentin $300 \mathrm{mg}$ in the morning and $600 \mathrm{mg}$ at night and tizanidine $2 \mathrm{mg}$ nightly for restless leg syndrome. Her headache day frequency prior to admission was four per month. She effectively used a combination analgesic pill containing butalbital-acetaminophen-caffeine four times per month. Triptans were contraindicated given history of cerebrovascular ischemic disease.

The patient's presenting symptoms were cough, followed by fever, generalized weakness, and headache. She then developed shortness of breath. Her headache exacerbation progressively worsened, located in the occipital and frontal regions and described as throbbing and tight. She had nausea without emesis, poor appetite, photophobia, and phonophobia.

This was representative of her typical migraine attacks with a new feature of neck stiffness. The headache did not respond to her usual effective acute therapy at home. By the time she was admitted to the hospital, her headache was unbearable. She also noted dysphagia to pills prior to admission which she had experienced with prior multiple sclerosis flares. This was suspected to be a pseudoexacerbation given that brain magnetic resonance imaging (MRI) with and without contrast only showed chronic lesions without new or acute enhancement.

During hospitalization, she experienced auditory hallucinations and displayed odd behaviors. This occurred in the setting of CNS active medications including intravenous diphenhydramine, promethazine, and prochlorperazine, as well as additional doses of gabapentin and first dose of lacosamide. Otherwise, she was alert and oriented although at times required prompting.

NSAIDs and steroids were not used for the treatment of headache given concern for potential worsening of COVID-19 symptoms. The patient had mild transaminitis which also initially precluded the use of valproic acid. Triptans and dihydroergotamines were contraindicated due to history of cerebrovascular ischemia. The patient's headache exacerbation was so severe compared to symptoms of pneumonia that neurology was asked to provide symptomatic treatment recommendations. The features of neck stiffness, auditory hallucinations, and odd behaviors, as well as refractoriness to treatment, all suggested a possible secondary headache disorder such as meningoencephalitis from SARS-CoV-2.

Rescue treatment with acetaminophen $1 \mathrm{~g}$ intravenously and either prochlorperazine, promethazine, or ondansetron co-administered with diphenhydramine given every $8 \mathrm{~h}$ were ineffective. She had a robust response to the initial administration of lacosamide $200 \mathrm{mg}$ intravenously, bringing her headache from a numeric rating of $10 / 10$ to $2 / 10$; however, this effect was not sustained and was not replicated with further dosing of lacosamide. Therefore, rapid titration of current and previously effective preventive medications was initiated. The patient's home medication gabapentin was increased while monitoring for signs of over-sedation. Topiramate was also re-initiated initially at $50 \mathrm{mg}$ twice daily (BID) with plan to increase to $100 \mathrm{mg}$ BID the following day. Fremanezumab $225 \mathrm{mg}$ was administered 2 weeks early. Lidocaine patch was applied to the back of the neck for pain and stiffness with some improvement. Opioids including morphine and hydromorphone were used sparingly and as a last resort given potential for respiratory compromise. COVID-19 pneumonia was treated with hydroxychloroquine and azithromycin. She had daily ECGs and continuous telemetry monitoring for QTc and PR prolongation.

The patient ultimately developed recurrent fevers and increasing oxygen requirements necessitating transfer to the intensive care unit and intubation. A lumbar puncture was not obtained to confirm or refute meningoencephalitis since it would not change management, and she had a brain MRI without new areas of enhancement including the leptomeninges. She continued to take lacosamide $200 \mathrm{mg}$ twice daily and gabapentin $900 \mathrm{mg}$ three times daily while in the intensive care unit and upon discharge from the hospital. One week post-hospitalization, her headache was resolved.

\section{Conclusions}

In a patient with confirmed or suspected COVID-19, the consulting neurologist should limit exposure if possible by performing either a phone or video consultation. It is clear that the specialty of neurology is needed during the pandemic by the wide range of neurologic manifestations of COVID-19, and many neurologic emergencies can be evaluated via teleneurology $[7,8]$. During consultation for headache in a patient with COVID-19, the primary goal should be to differentiate a secondary headache from a primary headache exacerbation. MRIs should be avoided unless absolutely necessary in COVID-19 patients due to the time it takes to decontaminate the MRI space after use. The use of teleneurology exams can be used to determine if focal neurologic symptoms are present [8]. It cannot, however, substitute a funduscopic exam, and if high suspicion for elevated intracranial pressure exists, imaging and lumbar puncture should be obtained. Otherwise, lumbar puncture should be pursued if it will change management. The number of reported cases of COVID-19 meningoencephalitis continues to grow, and SARS-2-CoV is not always isolated in the cerebrospinal fluid (CSF) [9]. Observationally, the presence of SARS-2-CoV does not seem to directly correlate with disease severity 
although it is too early to make such a distinction $[10,11]$. To date, one other case has reported intense headache as a symptom [9] but none of the meningoencephalitis cases were reported to have treatment-refractory headache or prior history of primary headache disorder.

For symptomatic treatment, the anti-convulsant class of medications may offer benefit with least risk of medication interactions and side effects. Careful attention to signs of hepatitis or kidney injury should be noted since severe cases of COVID-19 have multi-organ involvement [1]. Additionally, the CGRP monoclonal antibodies for migraine prevention may play an important role in reducing acute headache severity given relatively fast onset of action compared to other oral preventive medications. In this case, an early dose of fremanezumab $225 \mathrm{mg}$ was given and was felt to be safe since alternative dosing is $675 \mathrm{mg}$ every 3 months. It should also be noted that the initial management of patients with COVID-19 is likely to occur in the emergency department. Per American Headache Society guidelines, the medications with the highest level of evidence for the treatment of migraine in the emergency department are prochlorperazine, metoclopramide, and subcutaneous sumatriptan [12]. Though the consensus on the use of these medications refers to a primary headache disorder, in the early treatment of patients with COVID-19, a secondary headache may not be fully realized. Respiratory status, level of sedation, and QTc should be monitored during treatment due to co-administration of QTc prolonging medications such as hydroxychloroquine and cardiac sequelae of COVID-19 [13, 14]. As described in this case, rescue therapies may be ineffective and preventive therapies need to be optimized. A limitation to this case report is that CSF analysis was not performed; however, we are the first to report how to manage acute treatment-refractory headache in a patient with COVID19. Overall, this case speaks to the need for more objective data regarding the treatment of seriously ill patients with COVID-19.

Author Contributions KNA designed and conceptualized the manuscript, analyzed data pertinent to the manuscript, and provided intellectual content. AJS also conceptualized the manuscript and revised for intellectual content.

\section{Compliance with Ethical Standards}

Written informed consent was obtained from the patient for publication of this case report.

Conflict of Interest Dr. Arca has no disclosures or conflict of interest. Dr. Starling has the following disclosures: consulting fees-Alder, Amgen, Eli Lilly \& Company, Impel, Lundbeck, Novartis, Medscape.

Abbreviations COVID-19, Coronavirus disease 2019; SARS-2-CoV, Severe acute respiratory syndrome coronavirus 2 ; PCR, Polymerase chain reaction; NSAID, Non-steroidal anti-inflammatory drug; WHO, World Health Organization; CDC, Centers for Disease Control; NIH, National Institutes of Health; MRI, Magnetic resonance imaging; BID, Twice daily; CSF, Cerebrospinal fluid

\section{References}

1. Mao L, Jin H, Wang M, Hu Y, Chen S, He Q, Chang J, Hong C, Zhou Y, Wang D, Miao X, Li Y, Hu B (2020) Neurologic manifestations of hospitalized patients with coronavirus disease 2019 in Wuhan, China. JAMA Neurol https://doi.org/10.1001/jamaneurol. 2020.1127 .

2. CDC. Coronavirus disease 2019 (COVID-19). In: Cent. Dis. Control Prev; 2020. https://www.cdc.gov/coronavirus/2019-ncov/ hcp/clinical-tips-for-healthcare-providers.html.

3. @ olivierveran. \#COVID $19 \mid$ La prise d'anti-inflammatoires (ibuprofène, cortisone, ...) pourrait être un facteur d'aggravation de l'infection. En cas de fièvre, prenez du paracétamol. Si vous êtes déjà sous anti-inflammatoires ou en cas de doute, demandez conseil à votre médecin. https://twitter.com/olivierveran/status/ 1238776545398923264. Posted March 14, 2020.

4. Clinical management of severe acute respiratory infection when COVID-19 is suspected. https://www.who.int/publications-detail/ clinical-management-of-severe-acute-respiratory-infection-whennovel-coronavirus-(ncov)-infection-is-suspected. Accessed 14 Apr 2020.

5. CDC. Coronavirus disease 2019 (COVID-19). In: Cent. Dis. Control Prev; 2020. https://www.cdc.gov/coronavirus/2019-ncov/ hcp/faq.html.

6. National Institutes of Health (NIH) Concomitant Medications | Coronavirus Disease COVID-19. In: COVID-19 Treat. Guidel. https://www.covid19treatmentguidelines.nih.gov/concomitantmedications/. Accessed 22 Apr 2020.

7. Sellner J, Taba P, Öztürk S, Helbok R. The need for neurologists in the care of COVID-19 patients. Eur J Neurol n/a. 2020. https://doi. org/10.1111/ene.14257.

8. Majersik JJ, Reddy VK. Acute neurology during the COVID-19 pandemic: supporting the front line. Neurology. 2020;94:1055-7. https://doi.org/10.1212/WNL.0000000000009564.

9. Bernard-Valnet R, Pizzarotti B, Anichini A, et al. Two patients with acute meningo-encephalitis concomitant to SARS-CoV-2 infection. Eur J Neurol n/a. 2020. https://doi.org/10.1111/ene.14298.

10. Moriguchi T, Harii N, Goto J, Harada D, Sugawara H, Takamino J, et al. A first case of meningitis/encephalitis associated with SARS-Coronavirus-2. Int J Infect Dis IJID Off Publ Int Soc Infect Dis. 2020;94:55-8. https://doi.org/10. 1016/j.ijid.2020.03.062.

11. Yh H, D J, Jt H. SARS-CoV-2 detected in cerebrospinal fluid by PCR in a case of COVID-19 encephalitis: Brain. Behav. Immun; 2020. https://pubmed.ncbi.nlm.nih.gov/32387508/? from_single_result $=$ SARS-CoV-2+Detected + in + Cerebrospinal+Fluid+by+PCR+in+a+Case+of+COVID-19+ Encephalitis\&expanded search query $=$ SARS-CoV-2+ Detected+in+Cerebrospinal+Fluid+by+PCR+in+a+Case+of+ COVID-19+Encephalitis.

12. Orr SL, Friedman BW, Christie S, Minen MT, Bamford C, Kelley NE, et al. Management of adults with acute migraine in the emergency department: the American headache society evidence assessment of parenteral pharmacotherapies. Headache J Head Face Pain. 2016;56:911-40. https://doi. org/10.1111/head.12835.

13. Gupta R, Misra A. Contentious issues and evolving concepts in the clinical presentation and management of patients with COVID-19 infectionwith reference to use of therapeutic and 
other drugs used in co-morbid diseases (hypertension, diabetes etc). Diabetes Metab Syndr Clin Res Rev. 2020;14:251-4. https://doi.org/10.1016/j.dsx.2020.03.012.

14. Shao F, Xu S, Ma X, Xu Z, Lyu J, Ng M, et al. In-hospital cardiac arrest outcomes among patients with COVID-19 pneumonia in
Wuhan. China Resuscitation. 2020;151:18-23. https://doi.org/10. 1016/j.resuscitation.2020.04.005.

Publisher's Note Springer Nature remains neutral with regard to jurisdictional claims in published maps and institutional affiliations. 